\title{
Principles and Practice of Bioanalysis (2nd Edn.)
}

\author{
(R. F. Venn, ed., CRC Press, Taylor \& Francis Group, Boca Raton, \\ London, New York, 2008, 326 p., UK Pound Price 46.99)
}

DOI: $10.1134 / \mathrm{S} 0006297908120092$

The book consists of 15 chapters written by internationally distinguished experts from biotech and pharmacological companies, mostly located in United Kingdom.

Chapter 1 presents physicochemical properties of drugs and metabolites including their impact on results of bioanalysis.

Chapter 2 deals with sample preparation for bioanalysis. There are data about methods of protein precipitation, choice of solvents, types of extractions, and derivatization of compounds for bioanalysis.

Chapter 3 describes basic principles of theory and practice of HPLC, including types of columns, pumps, detectors, and other HPLC equipment. Analysis of various chromatographic parameters such as resolution, peak shape, and effects of temperature, flow rate, and sample volume are also discussed.

Chapter 4 considers various types of HPLC and optimization conditions for chromatography.

Chapter 5 deals with characterization of various types of HPLC detectors including UV-, fluoro-, radiochemical, optical, and other types of detectors.

Chapter 6 characterizes the basics of gas chromatography, sample preparation, injection types, and sample detection.

Chapter 7 deals with thin-layer chromatography including preparative approaches.

In chapter 8, immunological methods of bioanalysis are discussed. Here characterization of antibodies, meth- ods for antibody labeling, affinity chromatography, and advantages and disadvantage of immunoassays are considered.

Chapter 9 characterizes methods of preparation and automation of bioanalysis.

In chapters 10-12, there are descriptions of fundamental aspects of mass-spectrometry and application of this method for bioanalysis including qualitative and quantitative analysis of metabolites.

Chapter 13 includes descriptions of nuclear magnetic resonance methods and their application for elucidation of drug metabolism.

In chapter 14, there are data on metabolite isolation, including radiochemical techniques. There is discussion of solid-phase and liquid extractions, HPLC, and other methods.

Chapter 15 deals with strategy for the development of quantitative bioanalytical procedures. In special parts of this chapter there is discussion on standardization and method performance.

Each chapter contains a bibliography of the most important papers in each field discussed in the book. This book is quite valuable theoretically and methodologically for specialists of various fields of analytical chemistry and biochemistry, molecular biology, and biotechnology. I believe that this book will become an important desk book for researchers working with bioanalysis of various drugs and metabolites.
G. Ya. Wiederschain, Doctor of Biological Sciences 